(C) 1996 IEEE. Personal use of this material is permitted. However, permission to reprint/republish this material

for advertising or promotional purposes or for creating new collective works for resale or redistribution to servers

or lists, or to reuse any copyrighted component of this work in other works must be obtained from the IEEE.

\title{
Novosibirsk Tau-Charm Factory Design Study
}

\author{
N. Dikansky, V. Parkhomchuk, A. Skrinsky, V. Yakimenko, \\ Budker Institute of Nuclear Physics, Novosibirsk, 630090, Russia
}

\begin{abstract}
A method of colliding beams is developed to study the fundamental properties of matter in two directions. The first direction is aimed at achieving higher energies allowing the discovery of new particles and fields. The goal of the second one is the study of more delicate fundamental properties of quarks and fields by increasing luminosity in crudely studied energy ranges. The study of $\mathrm{CP}$ violation is the most famous example. To study the $\mathrm{CP}$ violation in the region of B-quarks, the project of a B-factory as an asymmetrical electron-positron collider with a beam energy of $7 / 4 \mathrm{GeV}$ and luminosity up to $10^{34} \mathrm{~cm}^{-2} \mathrm{~s}^{-1}$ was developed at BINP. During the development of this project, it became clear that the Institute's economical situation, which grew worse in the period of 1990-1993, did not allow us to carry out this project in a reasonable time. Besides, Japan (KEK) and USA (SLAC) began to build the B-factories. Therefore, it is hardly probable to obtain any new results on the third B-factory. On the other hand, a beam energy region of $0.7-2.5 \mathrm{GeV}$, which still is very interesting, is not studied completely. The interest in this field of research is due to a great body of information obtained on a LEAR facility which is utilized to study proton-antiproton annihilation approximately in this energy region. Studying the cross-section of production of observed resonances in electron-positron annihilation noticeably increases the value and reliability of their interpretation. In 1993, as a result of discussions, the International Program Committee on High-Energy Physics of the Ministry of Science recommended to reorientate the Novosibirsk project VEPP-5 into a Tau-Charm-factory with high luminosity [1].
\end{abstract}

\section{$\tau$-CHARM FACTORY}

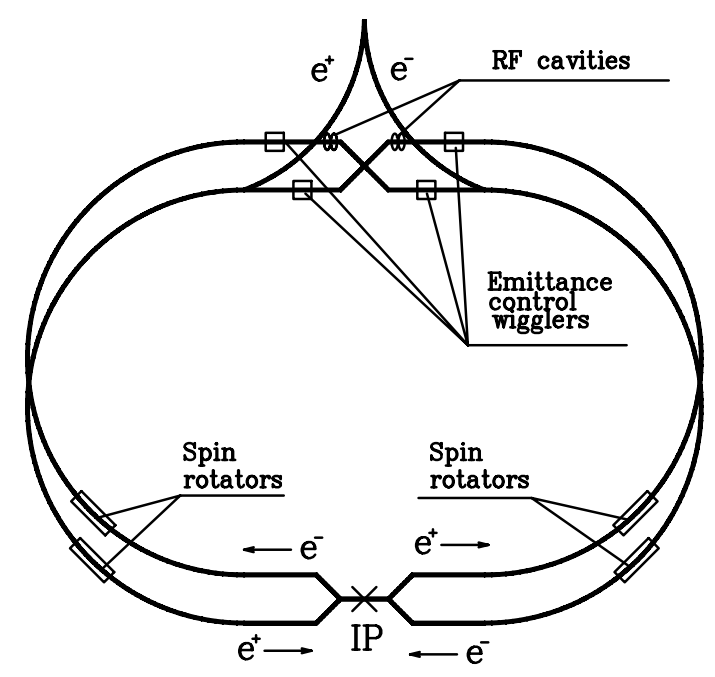

Figure. 1. Schematic layout

\section{Parameters of Colliding Beams and Interaction Region}

The beam parameters for the Tau-Charm-factory should satisfy the requirements for obtaining a maximum high luminosity of $1.010^{34} \mathrm{~cm}^{-2} \mathrm{~s}^{-1}$. In addition to the maximum luminosity regime, it is planned to obtain the beam parameters required for the so-called monochromatization of colliding beams and polarized colliding ones. To work in these regimes, the beam emittance control systems are needed. Thus, to obtain the monochromatic colliding beams, it is necessary that the main contribution to the vertical size be made by energy spread at the interaction point and the vertical betatron size be substantially less. To control and preserve polarization, rather long solenoids with a longitudinal magnetic field are required. It is undoubtedly impossible to provide simultaneously all these regimes; so, regimeto-regime transition will be made by replacing magnetic elements in straight sections. In this case, the contribution of half-rings into emittances should be as small as possible. The maximum luminosity of the facility with round beams is determined by the expression

$$
L_{\max }=\frac{c N_{\max } \gamma \xi_{\max }}{r_{e} D_{\min } \sigma_{l}},
$$

where $N_{\max }$ is the maximum number of particles in a bunch; $D_{\min }$ is the interbunch distance, $\xi_{\max }$ is the parameters of beam interaction; $r_{e}$ is the classical electron radius; $\gamma=E / m c^{2}$, and $\sigma_{l}$ is the bunch length (sigma). Assuming that a RF system is used at a $700 \mathrm{MHz}$ frequency and the interbunch distance is multiple to the RF wave length, we can present the beam parameters for the maximum luminosity in Table.1.

In the energy monochromatization regime, the value of a vertical energy dispersion is chosen according to the energy spread. In this case, the value of monochromatization is determined by the ratio of the vertical betatron size and the vertical energy one.

It is necessary to completely reconstruct the experimental straight section to obtain the ultra monochromatic colliding beams $\sigma_{M} \approx 6 \mathrm{keV}$. The strong quadrupoles and weak dipoles should be used for beam separation and control of the vertical dispersion function at the interection point. The length of such section is estimated to be more than $30 \mathrm{~m}$. The beam parameters in this case are presented in table:

\begin{tabular}{|l|l|}
\hline Number of particles in bunch & $1.510^{11}$ \\
\hline Interbunch distance $(\mathrm{m})$ & 16.28 \\
\hline Beta-function at IP $(\mathrm{cm}) \beta_{x} / \beta_{y}$ & $20 / 2$ \\
\hline The value of $\xi_{\max } / \xi_{D}$ & $0.05 / 0.01$ \\
\hline Beam emittance $\epsilon_{x} / \epsilon_{y}(\mathrm{~cm})$ & $10^{-5} / 10^{-9}$ \\
\hline Beam current $(\mathrm{A})$ & 0.4 \\
\hline Energy resolution $\sigma_{M}(\mathrm{keV})$ & 6 \\
\hline Luminosity $\left(\mathrm{cm}^{-2} \mathrm{~s}^{-1}\right)$ & $5.010^{31}$ \\
\hline
\end{tabular}

The Tau-Charm-factory is located in a tunnel with a $3 \times 3 \mathrm{~m}^{2}$ cross-section, whose floor and ceiling are 163 and $166 \mathrm{~m}$ above 


\begin{tabular}{|l|c|}
\hline Energy (Gev) & 2.1 \\
Circumference $(\mathrm{m})$ & 773.036 \\
Ring radius $(\mathrm{m})$ & 89.63 \\
Interbunch distance $(\mathrm{m})$ & 8.14 \\
Straight section lenght $(\mathrm{m})$ & 100 \\
Beam radius at IP $(\mu \mathrm{m})$ & 33 \\
Number of rings & 2 \\
Number of bunches per beam & 95 \\
Number of particle per bunch & $2 \times 10^{11}$ \\
b-function at IP $(\mathrm{cm})$ & 1 \\
Beams emittance $\left(\epsilon_{x}=\epsilon_{y}\right)(\mathrm{cm} / \mathrm{rad})$ & $10^{-5}$ \\
RMS bunch length $(\mathrm{cm})$ & 0.8 \\
Compaction factor & $0.001 \div 0.0017$ \\
Betatron tune $\nu_{x}$ & 29.077 \\
Betatron tune $\nu_{y}$ & 31.077 \\
Vertical damping time $(\mathrm{s})$ & 0.11 \\
RF voltage $(\mathrm{kV})$ & 1000 \\
RF frequency $(\mathrm{MHz})$ & 700 \\
Energy loss per turn $(\mathrm{keV})$ & 100 \\
Energy spread & $5 \times 10^{-4}$ \\
Harmonic Number & 1805 \\
Tune shift parameter $\xi_{x}=\xi_{y}$ & 0.1 \\
Design Luminosity $\left(\mathrm{cm}^{-2} s^{-1}\right)$ & $10^{34}$ \\
\hline
\end{tabular}

Table.1

the sea level, respectively. The underground room consists of two half-rings, $89.58 \mathrm{~m}$ in radius, and straight section $100 \mathrm{~m}$ long, which connects the half-rings. In this case, the length of an ideal orbit is $773.036 \mathrm{~m}$, corresponding to 1805 lengths of the RF wave. With such a geometry, each 19th separatrises contains a particle bunch; in all, the ring has 95 bunches. To install the injection equipment and magnetic systems of emittance control, the technical gap is increased up to $3 \times 5 \mathrm{~m}^{2}$. The gap should be widened to the side of injection channels so that to have $1.5 \mathrm{~m}$ to the inner wall and $3.5 \mathrm{~m}$ to the external one. The nearest-to-thesurface point of the tunnel is at a $10 \mathrm{~m}$ depth; the nearest-withinreach point is the bottom of a technological line, which is 168.6 $\mathrm{m}$ above the sea level, corresponding to a ground thickness of 2.6 $\mathrm{m}$ above the ceiling of the Tau-Charm-factory tunnel. At present, the tunnel, $200 \mathrm{~m}$ long, beginning from the injection complex to a shaft, where the injection channel of the Tau-Charm-factory begins, has been built. In the nearest future, we shall begin to build the channels distributing the electron and positron bunches into an injection gap. The factory is built by the firm "Gornyak" at a pace depending mainly on the financial possibilities. Keeping the pace, we shall need about 5 years to build the underground part.

\section{Element of Magnetic System Periodicity}

The Tau-Charm-factory magnetic system consists of two storage rings located over each other and intercrossing at the interaction point. To provide collisions of longitudinally polarized beams, spin rotators are supposed to be installed in the half-rings. As an example of the specific magnetic system, we present a simple system which consists of a dipole magnet, 1.5 $\mathrm{m}$ long, with a field of $1024 \mathrm{G}$ and quadrupole lenses, each has a $0.4 \mathrm{~m}$ length, whose parameters are given in detail in table :

\begin{tabular}{|l|l|l|l|}
\hline & Length $(\mathrm{cm})$ & Field $(\mathrm{kG})$ & Gradient $(\mathrm{kG} / \mathrm{cm})$ \\
\hline Quad & 40 & & 1.0631 \\
\hline Gap & 30 & & \\
\hline Dipole & 150 & 1.042 & \\
\hline Gap & 30 & & \\
\hline Quad & 40 & & -1.0631 \\
\hline Gap & 30 & & \\
\hline Dipole & 150 & 1.042 & \\
\hline Gap & 30 & & \\
\hline
\end{tabular}
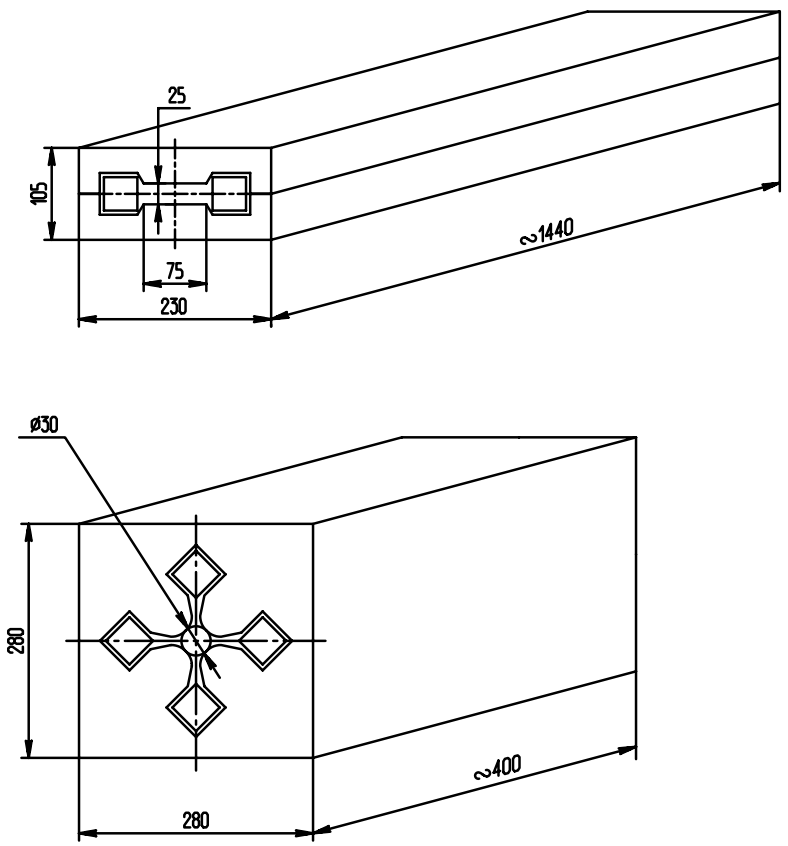

Figure. 2. Magnet elements of periodicity cell

\section{Emittance Control Section}

To control emittance, it is necessary to use special magnetic system sections where the magnetic field can be changed. A wellknown element used for this purpose is the wiggler-magnet which allows us to noticeably increase the energy loss for radiation. As a rule, the $\psi$-function is small in the wiggler-magnet. As a result, introducing strong decay, the magnet does not give a considerable increase in the emittance. Let us consider a special magnet design which gives a strong dispersion and the introducing of which will significantly increase the emittance. The equation for the $\psi$-function has the form

$$
\frac{d^{2} \psi}{d s^{2}}+G \psi=K
$$

In a smoothed approximation, in the case where the phase is assumed to be constant along the magnetic gap and the dipole magnetic field to be modulated by the resonance frequency, the amplitude of the $\psi$-function beats increases linearly. To obtain the necessary emittance under these conditions, the length of such a section should be several tens meters. At the point where $\psi$ is maximum, the wiggler-magnet controlling the emittance value in the given direction is placed. For two rings, the electron and positron ones, we need four sections with wiggler-magnets to independently tune the beam emittances. 


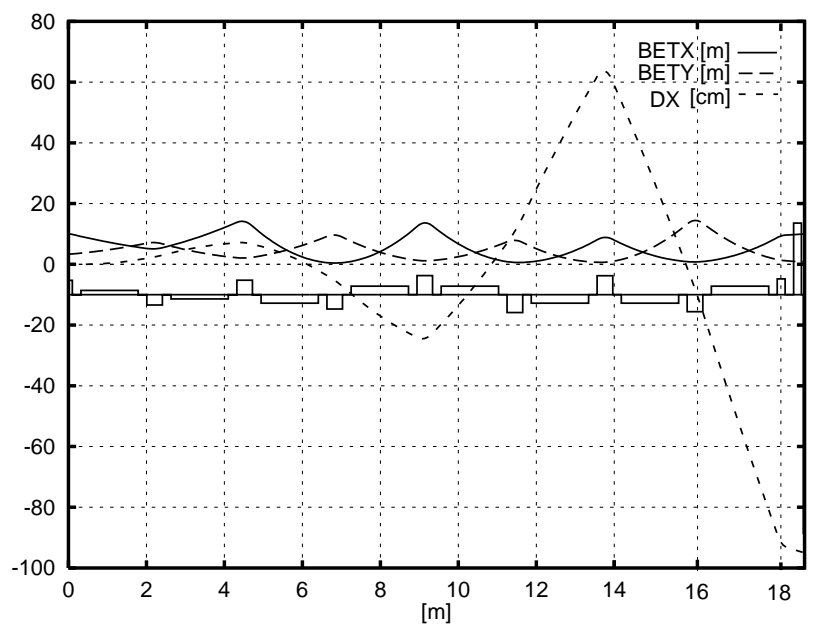

Figure. 3. Insertion of the $\psi$-function control

\section{Interaction Region}

The most interesting possibility of obtaining the maximum luminosity is the setting up of the interaction point with a small $\beta$-function using a strong longitudinal field (9.6 T and $2.18 \mathrm{~m}$ long). Possessing symmetrical focusing in both directions, such a system satisfies the idea of operation with round beams and can allow us to obtain the parameters for a space charge $\xi \geq$ 0.1 . The main problem in setting up the interaction point is a necessity of electrostatic beam separation. The total length of separation plates with a field of $100 \mathrm{kV} / \mathrm{cm}$ is $2 \mathrm{~m}$. They are located at the point where the $\beta$-function value is high, presenting the difficulties in providing the coherent beam stability. A steady obtaining of a powerful electrical field under the conditions of synchrotron irradiation of the separation plates is also a rather serious problem. It is planned to carry out experiments on VEPP$2 \mathrm{M}$ for producting on extremely high field strength under the synchrotron light.

\section{Conclusion}

The construction of the Tau-Charm-factory under the existing economic conditions in Russia is not a simple problem. However, the successful completion of this project will allow us to enter the third millenium with an interesting machine which the world scientific community needs to acquire fundamental knowledge.

\section{References}

[1] Dikansky N.S., et al. Status of the VEPP-5 Complex, Proc. of EPAC94, London, 1994, p.482 